\title{
Effects of Skin Surface Lipids on Skin Health
}

Faqing Feng ${ }^{1}$, Laiji $\mathrm{Ma}^{2}$, Zhaohui $\mathrm{Qu}^{3}$, Yinmao Dong ${ }^{1,2}$, Fan $\mathrm{Yi}^{1^{*}}$

${ }^{1}$ Key Laboratory of Cosmetic, China National Light Industry, Beijing Technology and Business University, Beijing, China

${ }^{2}$ Chinese Cosmetics Collaboration Innovation Center, Beijing Technology and Business University, Beijing, China

${ }^{3}$ Nutri-Woods Bio-Tech (Beijing) Co., Ltd. Beijing, China

\author{
"Corresponding author: Fan Yi, Key \\ Laboratory of Cosmetic, China National \\ Light Industry, Beijing Technology and \\ Business University, No.11/33, Fucheng \\ Road, Haidian District, Beijing, China \\ Tel.: +861068984937 \\ Email: fantasyee@btbu.edu.cn
}

Received January 23, 2019

Revised February 20, 2019

Accepted February 21, 2019

Published March 30, 2019

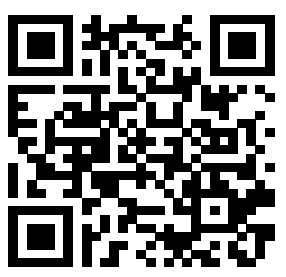

\begin{abstract}
Skin surface lipids are composed of sebaceous glands secreting lipids and epidermal disintegration lipids. The main components include triglycerides, free fatty acids, wax esters, squalene, cholesterol and so on. Skin surface lipids adhere to the surface of the skin and rely on their physical, chemical and biological properties to be important for skin health. This article reviews the relevant literatures at home and abroad, briefly describes the effects of skin surface lipids on skin moisturization, anti-oxidation and maintenance of micro-ecology, and mainly introduces the oxidation/metabolism of squalene/free fatty acids, which is more affected by external harmful factors than other skin surface lipids. As well as, the effects on skin health of squalene oxidation products and triglyceride metabolites are introduced. Above all, the oxidation/metabolism of skin surface lipids and the role of their products are illustrated in order to reveal the relationship between skin surface lipids and skin health and to provide a theoretical reference for the research and development of cosmetics by regulating skin surface lipids.
\end{abstract}

Keywords: Skin surface lipids, Squalene, Free fatty acids, Oxidation, Metabolism

\section{Introduction}

皮肤表面脂质（skin surface lipids, SSLs）是皮肤最外 层皮脂膜的主要成分, 其功能与脂质含量、成分、比例结构 密切相关。随着化妆品科学的发展和技术进步, 近年来针对 皮肤表面脂质、以及内、外环境因素引起的改变, 与皮肤健 康状态的关系成为研究热点。一方面, 目前对于正常SSLS的认 识已经比较清楚, 根据正常SSLs比例结构而构建的天然来源或 人工合成的SSLs类似物, 在化妆品中得到广泛应用并收到良好 效果 (Addy et al., 2017; Blaak et al., 2017; Oh et al., 2016) ; 另一方面, 人们也逐渐认识到, 正常皮肤分泌和代谢 的脂质, 在紫外线或空气污染物等外界有害因素的作用下, 发 生氧化、异常代谢等，其产物对皮肤产生损害 (Niki et al., 2015; Lohan et al., 2016; Shimizu et al., 2018)。

在外界环境有害因素作用下, 究竟有多少正常SSLS成分发生 变化, 对皮肤可能产生的损伤是什么, 还未见相应的系统报 道。本文在分析了SSLs与皮肤健康的关系的基础上, 并综述了
角鲨烯氧化、甘油三酯代谢, 及其对皮肤的危害, 为化妆品研 究人员从调节SSLS角度出发以维持皮肤健康状态提供思路。

\section{Skin surface lipids}

SSLs是由皮脂腺分泌脂质和脱落表皮崩解脂质组成的混合 物, 其组成和含量随皮脂腺分泌脂质含量及脱落的表皮细胞数 目不同而变化。皮脂腺来源的脂质由皮脂腺合成, 并以全浆分 泌的形式被排泄到皮肤表面; 表皮来源的脂质由角质形成细胞 合成, 是随着角质层细胞的代谢崩解分布至皮肤表面的结构脂 质 (Feingold et al.，2012)。SSLs与汗腺分泌的汗液及皮肤 表面透皮蒸发水, 融合乳化在皮肤表面形成一层膜, 通常称为 皮脂膜。

SSLs与人体其他内部组织器官有不同的脂质组成和代谢特 点。Nicolaides (1974) 对SSLs的组成 (见Table 1) 和作用进 行了广泛研究。 
Table1. The lipid composition of various sources of adult human skin

\begin{tabular}{lccc}
\hline Compositions & $\begin{array}{c}\text { Sebaceous gland lipid } \\
\text { (from sebaceous glands) }\end{array}$ & $\begin{array}{c}\text { Epidermal lipid } \\
\text { (from epidermal cells) }\end{array}$ & SSLs \\
Squalene & 12 & $<0.5$ & 10 \\
Wax esters & 23 & 0 & 22 \\
Triacyl glycerols & 60 & 10 & 25 \\
Di- and monoacyl glycerols & 0 & 10 & 10 \\
Sterol esters & $<1$ & 10 & 2.5 \\
Sterols & 0 & 20 & 1.5 \\
Free fatty acids & 0 & 10 & 25 \\
Glyco- and phospholipids & 0 & 30 & 0 \\
Others & 5 & 10 & 4 \\
\hline
\end{tabular}

SSLs, skin surface lipids.

\section{The function of skin surface lipids on skin}

SSLs依赖其物理、化学、生物特性对皮肤起保护作用, 以应 对外界刺激。SSLs主要通过润泽、抗氧化作用和保持皮肤微酸 性来维持皮肤健康(He et al., 2012)。

\section{Moisturizing}

SSLs利于保持皮肤水分, 使皮肤滑润柔韧、富有光泽。研究 表明, 相对于健康人, 以角质层水合作用减少、经皮水分散失 增强为特征的特应性皮炎人群的皮脂腺脂质（尤其是角鲨烯 和蜡酯) 含量减少2倍 (Shi et al., 2015)。Rissmann et al. (2012) 验证制备的胎儿皮脂类似物对皮肤有很好的保水和促进 皮肤屏障恢复作用。

\section{Anti-oxidation}

早期研究SSLs在 $215 \mathrm{~nm}$ 有最大光谱吸收, 并证实前额上的皮 脂膜会减少 $300 \mathrm{~nm}$ UV约 $10 \%$ 的透射率。并且, SSLs中的角鉴烯 (Boussouira et al., 2016)、甘油三酯 (Jin et al., 2014)等 通过不同分子机制, 结合或清除皮肤表面产生的活性物（如自 由基、炎性因子等）, 减少皮肤因化学应激、紫外辐照、微生 物代谢等引起的氧化、炎症损伤。

\section{Maintain micro-ecology}

SSLs中的游离脂肪酸 (free fatty acid, FFAs) 和汗液中的 酸性成分维持皮肤表面的微酸性环境(Man et al., 2007), 这 种微酸环境不仅利于皮肤形成化学屏障以应对外界酸、碱刺 激, 更重要的是利于特异性选择定植在皮肤上的微生物菌群。

皮肤表面定居大量的微生物, 主要为细菌, 少量真菌、病毒 和螨虫, 它们基于皮肤不同生理形态下的微环境, 组成生态系 统, 常称为 “皮肤微生态”, 其主要作用为消化凋亡细胞、分 解多余脂质、通过竞争性抑制抵抗外来病原菌入侵。已有研究 表明, 皮肤中的微生物一免疫相互作用通过产生关键的抗炎和 抗微生物物质(如抗菌肽, antibacterial peptide, AMP) 以
维持健康的组织平衡状态 (Sanford et al., 2013), 对于保持 皮肤最佳屏障功能、病原体防御和组织修复至关重要。

角质形成细胞参与皮肤免疫反应, 通过表达许多模式识别 受体 (pattern recognition receptors, PRRs), 通过识别 保守的分子实体如脂蛋白, 核酸, 细胞壁成分和鞭毛来感知 微生物。虽然角质形成细胞在稳态下表达许多抗微生物肽, 细胞因子和趋化因子, 但PRRs的激活可以迅速增加这些分子 的表达, 从而产生直接的抗菌作用以及额外免疫细胞的募集 和教育 (Sanford et al., 2013)。皮肤的共生细菌可产生具有 抗菌特性的分子以限制皮肤病原微生物的生长, Iwase et al. (2010) 表明表皮葡萄球菌的临床分离株能够通过产生丝氨酸蛋 白酶 $\mathrm{Esp}$ 来抑制金黄色葡萄球菌生物膜的形成, 并增强抗菌肽 (human- $\beta$-defensins, hBD-2) 的抗菌作用。皮肤微生物也 可通过影响宿主细胞的功能而促进皮肤免疫, 共生微生物通过 TLR2的信号传导导致角质形成细胞中抗微生物肽的表达增加, 并阻断由致病性金黄色葡萄球菌诱导的 NF- $\mathrm{kB}$ B抑制 (Wanke et al.，2011）。Plichta et al.（2017）研究确定表皮脂质和各 自的脂质合成酶在供体皮肤和烧伤边缘均显着降低, 并进一步 确定了供体皮肤和烧伤边缘中调节屏障通透性和AMP活性的表 皮蛋白酶的异常产生, 以及由AMP诱导的促炎细胞因子的更多 产生。

微酸性环境抑制皮肤表面微生物的生长, 尤其FFAs对皮肤微 生态平衡有重要影响。Nicolaides (1974) 早期预测FFAs决定 皮肤表面的微生物类型, Nakatsuji et al. (2010)证实FFAs 对痤疮丙酸杆菌有直接抗菌活性, 并通过诱导皮脂腺细胞中 $\mathrm{hBD}-2$ 的表达, 来增强皮肤的先天抗菌防御能力。

\section{Oxidation/metabolism of skin surface lipids}

SSLs不仅决定皮肤的屏障功能, 也是重要的信号传递介质。 环境压力改变SSLs组成、反应性和分布, 从而影响皮肤生物学 (Gruber et al.，2016)。目前, 由天然或医源性紫外线照射、 
常驻和致病微生物菌群的代谢活动、化学品和化妆品暴露所 引起的SSLs的定性修饰 (De Luca et al., 2010)一直是研究重 点。

\section{UV and air pollution affects the oxidation of skin surface lipids}

SSLs在介导环境刺激/压力源的生物效应方面的作用得到确 认 (Boussouira et al., 2016), 角鲨烯（squalene, SQ）因结 构中含 6 个双键成为其中最敏感的脂质成分。

外界刺激作用于皮肤, 一旦外界刺激突破人体抗氧化剂 $\alpha-$ 生 育酚和泛醇- 10 的防御, SQ将成为最活跃的牺牲型抗氧化剂, 其与单线态氧 $\left({ }^{1} \mathrm{O}_{2}\right)$ 反应, 发生高度复杂的氧化链反应, 生 成角鲨烯氧化物成为皮肤表面主要的脂质过氧化物, 包含大量

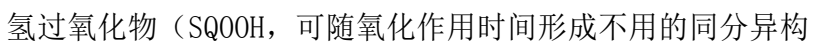
体），和少量氢氧化物（SQOH）（Ekanayake et al.，2003）。

紫外线和空气污染是影响皮肤氧化状态最常见且有效的因 素。紫外线 (主要是UVA) 通过作用于由丙酸杆菌在毛囊导管 中产生的卟啉, 产生多种形式的高活性氧原子 (主要是 ${ }^{1} 0_{2}$ ), 来催化加速皮肤表面的 SQ氧化。空气污染中多环芳烃、氮氧化 物、硫化物等均能促进 $\mathrm{SQ}$ 氧化, 研究发现香烟烟雾与UVA协同 作用使 $\mathrm{SQ}$ 氧化程度大大加重, 在UVA诱导下, 香烟烟雾增强皮 脂氧化作用约 3.5 倍, 而香烟的甲醇提取物增加了 23 倍的角鲨 烯氧化 (Pham et al., 2015)。

\section{Microorganisms affect the metabolism of skin surface lipids}

皮肤微生态的平衡维持皮肤的健康状态, 游离脂肪酸 (FFAs) 是其中主要的物质基础和重要的调控介质。FFAs在新 分泌的皮脂腺脂质中并不存在, 而在SSLs中含量约占 $25 \%$, 主 要是由皮肤表面定植的微生物产生的脂肪酶将皮脂腺脂质中的 甘油三酯水解得到, 因此皮肤表面微生物影响SSLs组成。FFAs 减少干燥、氧化等环境压力对皮肤的损伤, 同时为表皮微生物 生长提供营养物质和适宜的酸性环境, 抑制有害菌在皮肤的定 植和生长。FFAs的抗菌作用是皮肤菌落平衡的重要因素, FFAs 组成变化是皮肤微生物改变的主要原因（Takigawa et al., 2005)。

痤疮丙酸杆菌感染是痤疮主要的致病性触发因素, 这种亲 脂性细菌代谢产生大量FFAs, 引起慢性炎症反应和漏斗上皮 的纤维化, 从而维持黑头粉刺、恠疮和结节的形成, 有研究 者认为类似的机制部分解释特应性皮炎 (AD) 和脂溢性皮炎 (SD) 的慢性炎症过程 (Dawson et al., 2007)。Pappas et a1. (2013) 研究不同民族人群微生物菌群、FFAs组成与皮肤状 态的关系, 发现非洲裔美国女性皮肤微生物产生的FFAs中, 奇数碳链游离脂肪酸 $(\mathrm{C} 15: 0)$ 、( $\mathrm{C} 17: 1)$ 含量较低, ( $\mathrm{C} 14: 0)$ 、 $(C 16: 1 \triangle 10) 、(C 18: 1 \triangle 9)$ 含量较对照组高, 对应有更低的水 分散失 (TEWL) 和更好的皮肤屏障。

\section{Oxidation/metabolites of skin surface lipids cause damage to the skin}

皮肤脂质代谢具有高度活性和多样性, 脂质对于皮肤的屏障 和信号功能至关重要 (Kendall et al., 2015; van Smeden et a1.，2014）。SSLs的定性修饰，成为涉及皮脂代谢的皮肤病 学诊断的病理学标志, 如花斑癣、痤疮、特应性皮炎或脂溢性 皮炎等。

\section{SQ}

角鉴烯不会对皮肤结构和功能带来负面生理影响, 而角鲨烯 的氧化产物作用于更深的皮肤层, 介导局部防御性炎症、光保 护性免疫反应, 导致粉刺痤疮产生和加剧、并可能加速皮肤老 化, 在更高浓度可能诱导局部甚至全身性的免疫抑制, 甚至牵 涉到皮肤癌发生。已证实痤疮患者皮肤中SQ减少, 角鲨烯氧化 副产物增加, 且日光照射加剧痤疮症状。体外研究 $\mathrm{SQ}$ 氧化产物 对角质形成细胞的作用, Picardo et al. (1991) 发现, SQ氧 化产物在低浓度和短时间瞬育时刺激DNA和蛋白质合成, 而在 更高暴露时间和剂量下会诱导细胞损伤和抑制有丝分裂活性; 并且上调细胞促炎因子释放 (0ttaviani et al., 2006), 并可 能通过这种机制激活黑素细胞树突并刺激黑色素合成。

\section{FFAs}

FFAs 对维持和恢复皮肤屏障、保持皮肤微生态平衡至关重 要, 但含量异常的FFAs会降低皮肤屏障、利于有害菌侵入, 造 成皮肤损伤甚至病变。Cannavò et al. (2017) 研究发现银屑 病患者和健康组相比, 非皮损区和皮损区均显示更高的脂质含 量水平, 对应更差的屏障功能。针对花斑㿍研究发现, 在皮脂 含量高的区域马拉色菌大量生长并出现大的无色斑点, 可能与 含量异常的不饱和脂肪酸亚油酸 (C18:2) 引起马拉色菌脂质过 氧化物酶的氧化活性显著增加有关 (Nazzaro-Porro et al., 1986)。van Smeden et al. (2014) 测定过敏性湿疹 (AE) 患者 皮损区较非皮损区长链游离脂肪酸含量降低、不饱和脂肪酸含 量增加, Ní Raghallaigh et al. (2012) 研究也发现乳头状 红斑痤疮患者皮脂中长链饱和脂肪酸含量降低, 表明长链脂肪 酸、饱和脂肪酸可能更利于皮肤保持健康状态, 这可能与皮肤 微生态的平衡相关。

\section{Conclusion}

SSLs对皮肤状态有重要影响, 内、外环境因素引起的成分、 含量及结构变化影响皮肤状态, 其中SQ和FFAs因其独特性而备 受关注, 其自身及其氧化/代谢的过程产物与皮肤的健康存在 必然联系, 其含量能够表征皮肤的皮肤的健康状态, 随着检测 
手段的进步和皮肤脂质组学的研究的深入, 更清晰明了的揭示 皮肤脂质中各成分的作用及作用途径有很重要的研究价值, 依 据SQ和FFAs的结构及代谢特点可以更有针对性的开发化妆品, 尤其是与皮肤脂质成分相关的化妆品原料和配方的开发。

\section{1. $S Q$}

基于SQ的高度不饱和及其在皮脂腺脂质中特异性存在的特 点, SQ可作为生物标志物用于高度敏感地表征皮肤所经受的氧 化压力, SQOOH也已被广泛证明是皮肤对环境应激反应的关键 介质。因此在化妆品研发中可建立体外试验平台, 通过测定 $\mathrm{SQ}$ 氧化速率或 $\mathrm{SQOOH}$ 含量及生成速率, 来篮查能够预防或控制 $\mathrm{SQ}$ 氧化或减少其氧化产物损伤的活性成分或终产品。基于角渻烯 氧化过程, 可以从猝灭单线态氧、清除自由基以中止 $\mathrm{SQ}$ 氧化链 反应、分解 $\mathrm{SQOOH及}$ 及清除 $\mathrm{SQOOH}$ 诱导的过多自由基、细胞因子和 炎症因子等多种途径降低角鲨烯氧化所引起的皮肤持续氧化、 炎症损伤。

\section{FFAs}

皮肤亚健康或病变状态, 常伴随微生态菌群失衡及FFAs组 成、含量异常, 通过调控皮肤表面微生态及脂质组成来改善皮 肤的亚健康状态, 是化妆品研发的可能思路。虽然不同种族、 性别、饮食习惯等人群皮肤微生物和FFAs组成存在较大个体差 异, 但随着研究的深入, 预计可通过选择性的添加相应链长/ 不饱和度的FFAs或其他有特异性抑菌作用的活性成分来定向抑 制致病菌的生长、维护皮肤微生态平衡, 从而增强皮肤屏障保 持皮肤健康状态。

\section{References}

Addy J, Oliphant T, Harper R. A botanically derived skin surface lipid mimetic based on the composition of healthy 22-year-old females. Journal of Cosmetic Science, 68: 59-67, 2017.

Blaak J, Dähnhardt D, Dähnhardt-Pfeiffer S, Bielfeldt S, Wihelm KP, Wohlfart R, Staib P. A plant oil-containing $\mathrm{pH}$ 4 emulsion improves epidermal barrier structure and enhances ceramide levels in aged skin. International Journal of Cosmetic Science, 39: 284-291, 2017.

Boussouira B, Pham DM. Squalene and skin barrier function: from molecular target to biomarker of environmental exposure. In: skin stress response pathways. Wondrak G (ed.), Springer, Switzerland, pp29-48, 2016.

Cannavò SP, Guarneri F, Giuffrida R, Aragona E, Guarneri C.
Evaluation of cutaneous surface parameters in psoriatic patients. Skin Research and Technology, 23: 41-47, 2017.

Dawson TL Jr. Malassezia globosa and restricta: breakthrough understanding of the etiology and treatment of dandruff and seborrheic dermatitis through whole-genome analysis. Journal of Investigative Dermatology Symposium Proceedings, 12: 15-19, 2007.

De Luca C, Valacchi G. Surface lipids as multifunctional mediators of skin responses to environmental stimuli. Mediators of Inflammation, 2010: 321494, 2010.

Ekanayake Mudiyanselage S, Hamburger M, Elsner P, Thiele JJ. Ultraviolet $A$ induces generation of squalene monohydroperoxide isomers in human sebum and skin surface lipids in vitro and in vivo. Journal of Investigative Dermatology, 120: 915-922, 2003.

Feingold KR. Lamellar bodies: the key to cutaneous barrier function. Journal of Investigative Dermatology, 132: 1951-1953, 2012.

Gruber F. The skin lipidome under environmental stress: technological platforms, molecular pathways and translational opportunities. In: skin stress response pathways. Wondrak G (ed.), Springer, Switzerland, pp128, 2016.

He CD, Liu WD, Zhang XJ. The foundation of modern dermatology. People's Medical Publishing House, Beijing, pp285-287, 2010.

Iwase T, Uehara Y, Shinji H, Taijima A, Seo H, Takada K, Agata T, Mizunoe Y. Staphylococcus epidermidis Esp inhibits Staphylococcus aureus biofilm formation and nasal colonization. Nature, 465: 346-349, 2010.

Jin XJ, Cheng S, Chen Z, Kim EJ, Chung JH, Yang XM. Effects of triglycerides metabolism on HaCaT cells after ultraviolet radiation and its possible mechanism. Progress in Modern Biomedicine, 14: 6454-6459, 2014.

Kendall AC, Pilkington SM, Massey KA, Sassano G, Rhodes LE, Nicolaou A. Distribution of bioactive lipid mediators in human skin. Journal of Investigative Dermatology, 135: 1510-1520, 2015.

Lohan SB, Müller R, Albrecht S, Mink K, Tscherch K, Ismaeel F, Lademann J, Rohn S, Meinke MC. Free radicals induced by sunlight in different spectral regions: in vivo versus ex vivo study. Experimental Dermatology, 25: 
380-385, 2016.

Man MQ, Xin SJ, Elias PM. Skin surface pH and its clinical implication. The Chinese Journal of Dermatovenereology, 21: 503-505, 2007.

Nakatsuji T, Kao MC, Zhang L, Zouboulis CC, Gallo RL, Huang CM. Sebum free fatty acids enhance the innate immune defense of human sebocytes by upregulating beta-defensin-2 expression. Journal of Investigative Dermatology, 130: 985-994, 2010.

Nazzaro-Porro M, Passi S, Picardo M, Mercantini R, Breathnach AS. Lipoxygenase activity of Pityrosporum in vitro and in vivo. Journal of Investigative Dermatology, 87: 108-12, 1986.

Ní Raghallaigh S, Bender K, Lacey N, Brennan L, Powell FC. The fatty acid profile of the skin surface lipid layer in papulopustular rosacea. British Journal of Dermatology, 166: 279-287, 2012.

Niki E. Lipid oxidation in the skin. Free Radical Research, 49: 827-834, 2015.

Nicolaides N. Skin lipids their biochemical uniqueness. Science, 186: 19-26, 1974.

Oh MJ, Nam JJ, Lee EO, Kim JW, Park CS. A synthetic C16 omega-hydroxyphytoceramide improves skin barrier functions from diversely perturbed epidermal conditions. Archives of Dermatological Research, 308: 563-574, 2016.

Ottaviani M, Alestas T, Flori E, Mastrofrancesco A, Zouboulis CC, Picardo M. Peroxidated squalene induces the production of inflammatory mediators in $\mathrm{HaCaT}$ keratinocytes: a possible role in acne vulgaris. Journal of Investigative Dermatology, 126: 2430-2437, 2006.

Pappas A, Fantasia J, Chen T. Age and ethnic variations in sebaceous lipids. Dermato-endocrinology, 5: 319-324, 2013.

Pham DM, Boussouira B, Moyal D, Nguyen QL. Oxidization of squalene, a human skin lipid: a new and reliable marker of environmental pollution studies. International Journal of Cosmetic Science, 37: 357-365, 2015.

Picardo M, Zompetta C, De Luca C, Cirone M, Faggioni A, Nazzaro-Porro M, Passi S, Prota G. Role of skin surface lipids in UV-induced epidermal cell changes. Archives of Dermatological Research, 283: 191-197, 1991.

Plichta JK, Holmes CJ, Gamelli RL, Radek KA. Local burn injury promotes defects in the epidermal lipid and antimicrobial peptide barriers in human autograft skin and burn margin: implications for burn wound healing and graft survival. Journal of Burn Care \& Research, 38: 212-226, 2017.

Rissmann R, Oudshoorn MH, Kocks E, Hennink WE, Ponec M, Bouwstra JA. Lanolin-derived lipid mixtures mimic closely the lipid composition and organization of vernix caseosa lipids. Biochimica et Biophysica ActaBiomembranes, 1778: 2350-2360, 2008.

Shimizu N, Ito J, Kato S, Otoki Y, Goto M, Eitsuka T, Miyazawa T, Nakagawa K. Oxidation of squalene by singlet oxygen and free radical results in different compositions of squalene monohydroperoxide isomers. Scientific Reports, 8: 9116, 2018.

Shi VY, Leo M, Hassoun L, Chahal DS, Maibach HI, Sivamani RK. Role of sebaceous glands in inflammatory dermatoses. Journal of the American Academy of Dermatology, 73: 856-863, 2015.

Sanford JA, Gallo RL. Functions of the skin microbiota in health and disease. Seminars in Immunology, 25: 370377, 2013.

Takigawa H, Nakagawa H, Kuzukawa M, Mori H, Imokawa G. Deficient production of hexadecenoic acid in the skin is associated in part with the vulnerability of atopic dermatitis patients to colonization by Staphylococcus aureus. Dermatology, 211: 240-248, 2005.

van Smeden J, Janssens M, Kaye EC, Caspers PJ, Lavrijsen AP, Vreeken RJ, Bouwstra A. The importance of free fatty acid chain length for the skin barrier function in atopic eczema patients. Experimental Dermatology, 23: 45-52, 2014.

Wanke I, Steffen H, Christ C, Krismer B, Götz F, Peschel A, Schaller M, Schittek B. Skin commensals amplify the innate immune response to pathogens by activation of distinct signaling pathways. Journal of Investigative, 131: 382-390, 2011. 


\section{中文摘要}

\section{皮肤表面脂质与皮肤健康的关系}

冯法晴 ${ }^{1}$, 马来记 ${ }^{2}$, 曲召辉 ${ }^{3}$ 董银卯 ${ }^{1,2}$, 易帆 $^{1^{*}}$

${ }^{1}$ 北京工商大学中国轻工业化妆品重点实验室，北京，中国

${ }^{2}$ 北京工商大学中国化妆品协同创新中心, 北京, 中国

3北京东方沝森生物科技有限公司，北京，中国

皮肤表面脂质是由皮脂腺分泌脂质和脱落表皮崩解脂质组成, 主要成分包括甘油三酯、游离脂肪酸、蜡酯、角 鲨烯、胆固醇等。皮肤表面脂质附着于皮肤表面，依赖其物理、化学及生物特性对皮肤健康具有重要的意义。 本文综述了国内外相关文献，简述了皮肤表面脂质对皮肤的润泽、抗氧化和维护微生态的作用，并主要介绍皮 肤表面脂质中受外界有害因素影响较明显的角鲨烯/游离脂肪酸的氧化/代谢过程, 以及产物如角鲨烯氧化产物、 甘油三酯代谢物游离脂肪酸等对皮肤健康的影响，来说明皮肤表面脂质的氧化/代谢过程及产物的作用，以期揭 示皮肤表面脂质对皮肤健康的关系，为调节皮皮表面脂质的化妆品的开发提供理论参考。

关键词: 皮肤表面脂质，角鲨烯，甘油三酯，氧化，代谢 


\section{국문초록}

\section{피부 표면 지질과 피부 건강과의 관계}

풍법청 ${ }^{1}$, 마래기 ${ }^{2}$, 곡조휘 ${ }^{3}$, 동은묘 ${ }^{1,2}$, 역범 $^{1 *}$

${ }^{1}$ 북경공상대학중국경공업화장품중점연구실, 북경, 중국

${ }^{2}$ 북경공상대학중국화장품공동혁신센터, 북경, 중국

${ }^{3}$ Netri-Woods 바이오테크닉, 북경, 중국

피부 표면 지질은 지질과 표피 붕괴 지질을 분비하는 피지선으로 구성됩니다. 주성분에는 트리글리 세라이드, 유리 지방산, 왁스 에 스테르, 스쿠알렌, 콜레스테롤 등이 포함됩니다. 피부 표면의 지질은 피부의 표면에 달라 붙어서 물리적, 화학적 및 생물학적 특성 에 의존하여 피부 건강에 중요합니다. 본 연구는 관련 문헌을 국내외에서 검토하고 피부 표면 지질이 피부 보습, 항산화 및 미세 생 태계 유지에 미치는 영향을 간략하게 설명하고 주로 다른 피부 표면 지질보다 외부 유해 요인의 영향을 더 받는 스쿠알렌 및 유리 지방산의 산화 및 신진 대사를 소개합니다. 뿐만 아니라, 스쿠알렌 산화 생성물과 트리글리세리드 대사 산물의 피부 건강에 미치는 영향에 대해 논술하였다. 무엇보다도 피부 표면 지질과 피부 건강 간의 관계를 밝히고 피부 표면을 조절하여 화장품의 연구 개발에 대한 이론적인 참고서를 제공하기 위함이다.

핵심어: 피부 표면 지질, 스쿠알렌, 유리 지방산, 산화, 대사 
